\title{
SCHWERPUNKT: PERFEKTIONISMUS
}

Was in akademischen Debatten gerade diskutiert wird, hat oft nur eine recht „lose Kopplung“ (Niklas Luhmann) mit dem, was in der Welt passiert. Die durch Gruppeneffekte und Reputationsgelüste mitangetriebene Themenwahl ist auch von äußeren Zufällen und persönlichen Vorlieben abhängig. Zusätzlich wird die Behandlung eines Themas oft noch dadurch verengt, dass sie durch die Brille einiger weniger Autoren gesehen wird, die gerade das Glück haben, gelesen zu werden. Die Philosophie kann auf diese Weise in eine Phase der kleinteiligen Exegese und dogmatischen Scholastik eintreten, was von außen - nicht ganz zu Unrecht als selbstgewählter „Elfenbeinturm“ wahrgenommen wird. Sachlich empfiehlt es sich daher wenig, die Bedeutung einer philosophischen Thematik primär am Stand einer, gegenwärtigen Debatte' festzumachen - zumindest dann nicht, wenn die Sache selbst seit Jahrhunderten diskutiert wird und auch politisch relevant ist. Das ist beim seltsam klingenden Perfektionismus als Strömung der moralischen und politischen Philosophie der Fall, wenn auch nicht immer unter diesem Titel (in der Tat handelt es sich eigentlich um einen ,,misnomer ${ }^{66}$ ). Daher soll hier zunächst angerissen werden, worum es in der Sache geht. Nur so nämlich werden blinde Flecken im aktuellen Stand der Debatte deutlich, die in diesem Schwerpunkt adressiert werden sollen.

Zunächst ein Wort zum irreführenden Namen. Es geht um eine Theorie des menschlichen Guten, die dieses als glücksförderliche menschliche Entwicklung begreift und auch zum normativen Maßstab sozialer Institutionen machen kann. Perfektionismus ist insofern ein ungünstiger Titel dafür, als dies suggeriert, es ginge darum, Menschen ,,perfekt“" zu machen. ${ }^{1}$ Falsch ist das insbesondere, wenn es religiöse Untertöne bekommt. Bei Aristoteles, dem zentralen Ideengeber eines nicht-totalitären Perfektionismus, besteht die „Vollkommenheit“" des Glückes lediglich darin, das dem Menschen Mögliche zu erreichen. ${ }^{2}$ Die menschliche Endlichkeit wird nicht zu überschreiten versucht, vielmehr wird das Streben auf erreichbare Ziele verwiesen (etwa: weg vom unendlichen Streben nach Reichtum, hin zu persönlichen Beziehungen). Erst dass die konfliktgeladene Aristoteles-Rezeption des Christentums versuchte, das menschliche Glück als etwas Jenseitiges zu begreifen, verlieh diesem Mischprodukt seine scheinbare Hybris. Doch sich selbst gottähnlich machen zu wollen, macht weder aus Aristoteles' Perspektive Sinn, da es ein unerreichbares Ziel wäre, noch - will man nicht pagan-magischen Praxen das Wort reden - aus christlicher Sicht: Keine Werkgerechtigkeit der Welt könnte dies vor Gott vollbringen. Christen können hoffen, dereinst erhöht zu werden,

1 „Not perfection as a final goal, but the ever-enduring process of perfecting, maturing, refining is the aim in living." (J. Dewey, Reconstruction in Philosophy [1920], New York ${ }^{2} 1950,141$ ) R. Kraut (What is Good and Why, Cambridge/Mass. 2007, 136) urteilt: „nothing can be perfect“, und schlägt für die Ethik des „flourishing“ den Namen „developmentalism“ vor. Eine gute Idee, doch wie soll man übersetzen: Bildungshumanismus?

2 Glück als höchstes menschliches Gut genügt sich selbst; vgl. Aristoteles, Nikomachische Ethik, 1094 b 7, 1097 b 7 ff., 1102 a 15. 
aber es nicht aus eigener Kraft erzwingen wollen. ${ }^{3}$ Darum sei betont: Der Perfektionismus will Menschen nicht ,perfekt" machen. Es geht ihm darum, Menschen zu ermuntern, sich und ihre Institutionen zu entwickeln und glücksförderliche praktische Ziele zu erreichen. Aristoteles begreift das als endliche Praxis, da die Möglichkeiten des Menschen endlich sind; der Marquis de Condorcet begriff es als unabschließbare Praxis, weil die Verbesserungsmöglichkeiten der Erzeugnisse des Menschen unendlich sind. Liest man eines als Aussage über die erste, das andere als Aussage über die zweite Natur des Menschen, ist das nicht einmal ein Widerspruch.

So viel zum Namen. Um den Perfektionismus näher zu verorten, seien zwei sehr grundsätzliche Eigenschaften der Menschen vorausgeschickt: Erstens, Menschen haben zwar eine bestimmte - allen ungefähr gemeinsame - natürliche Ausstattung, kommen mit ihrer Geburt aber nicht ,fertig' auf die Welt. Sie müssen sich erst entwickeln, aber in welche Richtung das geht, ist nicht festgelegt. ${ }^{4}$ Merkmal ihrer natürlichen Ausstattung ist vielmehr eine ungeheure Plastizität und Vielseitigkeit. Diese elliptische Aussage kann auf zwei Weisen verfehlt werden: Falsch wäre sowohl die Folgerung, der Mensch sei durch seine Natur auf bestimmte Weisen zu leben festgelegt (das wäre ein Naturalismus), als auch die, seine Entwicklung sei beliebig (das wäre ein Voluntarismus). Der perfektionistische Ansatz geht einen Mittelweg, indem verschiedene Weisen der Entwicklung verglichen und bewertet werden, um dann zu beurteilen, welche Wege gute Entwicklungswege wären. „Gut“ im perfektionistischen Sinn ist nicht einfach Entwicklung schlechthin, sondern gesucht werden - gerade angesichts der Vielfalt an Entwicklungswegen, aber auch an Leiden unter versäumter oder verhinderter Entwicklung - Kriterien einer guten Entwicklung des einzelnen Menschen.

Zweitens kann ein Einzelner, gerade weil er/sie unfertig geboren wird, eine solche Entwicklung nicht allein unternehmen (,,it takes a village to raise a child“). Menschen sind nolens volens soziale Wesen - selbst Misanthropen haben diese ihre Eigenschaft in Bezug auf andere Menschen. Aus diesem Grund sind in perfektionistischen Theorien Ethik und Politik meist enger verklammert als in neueren liberalen Theorien. Nicht nur, weil das Leben in gelingender Gemeinschaft selbst etwas „Gutes“ ist - das wäre eine instrumentalistische Verkürzung, welche liberale Alarmlampen anwirft, weil sie leicht Unterordnung legitimieren kann (siehe den Beitrag von Michael Schefczyk). Sondern: Wenn Individuen eine gute Entwicklung nehmen wollen und sollen, es aber unmöglich allein können, ist es gut, wenn die Gemeinschaft die Individuen dabei unterstützt. Daher ist auch diejenige Gemeinschaft „gut", in der sich die Individuen gut entwickeln können. Eine solche Gemeinschaft, die an der Entwicklung seiner Mitglieder interessiert ist, muss allerdings eine Vorstellung davon haben, was eine gute Entwicklung ist und was die entsprechenden Mittel wären, die ein Individuum beziehungsweise die Gesellschaft dafür braucht. Der Perfektionismus ist als Versuch einer Antwort auf diese Frage zu verstehen. Er hat daher von vornherein einen institutionellen und interessierten

3 Vgl. A. Rüdiger, Produktive Negativität. Die Rolle des Perfektionismus im deutschen Aufklärungsdenken zwischen Pufendorf und Kant, in: Deutsche Zeitschrift für Philosophie, 58 (2010) (vorliegendes Heft), Abschnitt II; vgl. ferner J. Passmore, The Perfectibility of Man, New York 1970, Kapitel 4-7. Wo es im antiken Christentum Selbstvergöttlichungskonzepte gab (etwa Origines' theosis), führten diese eher in die Mystik als in den hier interessierenden ethischen Perfektionismus.

4 A. Rüdiger (Produktive Negativität, a. a. O.) legt A. Gehlens Standpunkt, „der Mensch“ sei das nicht festgestellte Tier (ähnlich schon Nietzsche und Herder), schon im 17. Jahrhundert bei Pufendorf frei und zeigt seine Verknüpfung mit dem Gedanken der menschlichen Perfektibilität. Dass es dem Perfektionismus um ,the development of human nature“ geht, stellt auch T. Hurka (Perfectionism, Oxford 1993, 3) fest. 
Blick auf die Menschen. ${ }^{5}$ Daher wird er besonders relevant, wenn es darum geht, politische Gemeinschaften auf- oder umzubauen: in Aufklärung und Progressivismus ebenso wie heute, nach Jahrzehnten einer kulturellen und sozialen Trockenlegung.

Die neuere liberale Trennung in einen gefährlichen „Staatsperfektionismus ${ }^{* 6}$ und harmlose „Theorien des Guten“ für den Privatbereich ist für den älteren Perfektionismus daher in gewisser Weise künstlich. Da er nicht in dieses Raster passt, geriet er für den Liberalismus eine Weile aus dem Blickfeld. Den Perfektionismus nun, da er in die Diskussion zurückkehrt, in einer ausdifferenzierten Wissenschaftslandschaft unterzubringen, ist nicht einfach, denn er beinhaltet mindestens drei heute als unverträglich geltende Elemente: Theorien über den Menschen als Naturwesen (3), Theorien über die Gesellschaft (2) und normative Theorien (1). Damit ist bereits benannt, warum der Perfektionismus im heutigen Philosophieren, sofern es vom amerikanischen Liberalismus in der Folge von J. Rawls geprägt ist, große Widerstände überwinden muss. Diese betreffen alle drei Dimensionen:

Innerhalb des normativen Denkens (1) ist angesichts der kantischen Verengung der Moral auf Pflichten schon die Rede von einer „Normativität“ des Prudentiellen ungewöhnlich. Normativ meint allerdings nicht automatisch moralisch. Eine Klugheit, die das Gelingen oder Misslingen des ganzen Lebens oder einer Gemeinschaft betreffen kann, ist nicht einfach vom Tisch zu wischen wie beliebige Ratgeberliteratur. Hier haben neuere Theorien des ,guten Lebens“ inzwischen eine Lücke gefüllt, soweit es um individuelle Belange geht. ${ }^{7}$ Doch dem Perfektionismus geht es nicht nur um private Belange, und genau das bringt ihm die Kritik liberaler Philosophien ein - unterläuft er damit doch die rest-platonische Aufteilung der normativen Welt in privat und öffentlich, in das inhaltlich/materiale Gute und das formal/prozedurale Gerechte. Wer Vorstellungen vom Guten zur Grundlage von Politik mache, so heißt es, verlasse das Gebot staatlicher Neutralität und sei des Paternalismus verdächtig. Dieser Verdacht ist in Fällen berechtigt, wo bestimmte Lebensformen gegen den Willen der Bürger vorgeschrieben werden („legislating morality“). Doch das tun auch andere Richtungen (so wird zum Beispiel „,im Namen des Liberalismus“ eine bestimme Arbeitsethik verordnet) ${ }^{8}$, während es für den

5 Das sollte im Sinne von R. Geuss sein (vgl. ders., Realismus, Wunschdenken, Utopie, in: Deutsche Zeitschrift für Philosophie, 58 (2010), 419-429). M. Foucault (Geschichte der Gouvernementalität, 2 Bde., Frankfurt/M. 2004) legt dieses Moment an der „Policeywissenschaft“ und am Liberalismus im Modus der ,Entlarvung' frei - und erneuert so auf Umwegen das marxistische Credo, dass auch der Liberalismus Menschen lenken will (vgl. bereits E. Köhler, Arme und Irre. Die liberale Fürsorgepolitik des Bürgertums, Berlin 1977). Der institutionelle Blick erklärt, wie natürliche Gleichheit und individuelle Verschiedenheit zugleich behauptet werden können: Aus dem Teleskopblick der Institutionen sind alle Personen zwar je einmalig, doch gibt es von ihrer Natur her keine Unterschiede, die eine ungleiche Behandlung rechtfertigen könnte - keine Stände, Klassen, Rassen etc. Das war für J. Locke noch nicht selbstverständlich (vgl. W. Lepenies, Soziologische Anthropologie. Materialien, Frankfurt/M. 1977, 68).

6 Vgl. W. Kymlicka, Contemporary Political Philosophy: An Introduction, Oxford 22002, 248 u. 277; zur Unterscheidung zwischen ethischem und politischem Perfektionismus vgl. M. Schefczyk, Perfektionismus und Pathologien der Selbstverwirklichung, in: Deutsche Zeitschrift für Philosophie, 58 (2010) (vorliegendes Heft); sowie ferner: D. Rassmusen u. D. J. Den Uyl, Norms of Liberty. A Perfectionist Basis for Non-Perfectionist Politics, University Park/Penn State 2005.

7 Im Überblick: D. Fenner, Das gute Leben, Stuttgart 2007. Im Sinne Kants formulieren Klugheitsethiken für das Privatleben ,hypothetische Imperative“ (etwa: ,Wenn du gern Freunde finden möchtest, dann solltest du dir bessere Manieren angewöhnen'). Freundschaften mögen zum guten Leben gehören, aber ob und wie jemand neue Freunde finden möchte, bleibt ihm freigestellt. Problematisch wäre es erst, wenn bestimmte Manieren mit solchen Imperativen verbindlich gemacht würden.

8 D. King, In the Name of Liberalism: Illiberal Social Policy in the USA and Britain, Oxford 1999. 
Perfektionismus nur das letzte Mittel ist - es geht auch sanfter, etwa durch Bereitstellung der Mittel ohne Zwang („Nudge“). Er hat auf diesen Vorwurf daher gewichtige Antworten:

Zunächst ist ein Selbstmissverständnis der Philosophie zu vermuten: Im politischen Alltag mögen Formalismus, Prozeduralismus und Neutralität des Staates wünschenswert sein, etwa wenn es um die kulturelle Selbstbestimmung von Minderheiten geht. Aber ist die Philosophie „der Staat"? Werden diese Prinzipien aus der Politik in die philosophische Reflexion auf Politik übernommen, lösen sie deren Bezug auf brennende Themen. So hat sich die politische Philosophie des Liberalismus in den letzten Jahrzehnten durch ihre selbstgewählte Inhaltsferne etwas in die Isolation gebracht. Vielleicht bietet der Perfektionismus, verstanden als Theorie, die das Gute als gelingende menschliche Entwicklung begreift, einen Ausweg aus dem Elfenbeinturm: Er vermag einer emanzipatorischen Philosophie deutlichere inhaltliche Konturen zu geben, ohne dabei - wie bei den Absetzungsbewegungen Konservatismus, Sozialismus oder Kommunitarismus zu beargwöhnen war-das zentrale Prinzip des Liberalismus, die individuelle Selbstbestimmung, zu untergraben. Daher feierte er insbesondere in der angelsächsischen Philosophie eine kleine Renaissance. ${ }^{9}$

Der Perfektionismus kann nun versuchen, sich dem Neutralitätsprinzip anzuschmiegen. ${ }^{10}$ Er kann aber auch mutiger werden: So ließe sich die Vorstellung, es gebe klinisch ,reine', von den Niederungen der realen Welt unbefleckte Regionen des „Gerechten“ (zu denen die liberale Philosophie bezeichnenderweise Zuflucht nimmt, um ihre Prinzipien zu erlangen - um nur an Rawls' Urzustand und R. Dworkins fiktive Insel zu erinnern), als entweder weltfremd oder ideologisch zurückweisen. ${ }^{11}$ Was wäre für etablierte Vertreter mächtiger Interessen bequemer, als die eigenen Inhalte und Methoden als neutral und gerecht hinstellen zu können? Das heißt nicht, dass ,neutrale 'Verfahren nicht prinzipiell denkbar wären. Doch können sich Machtverhältnisse jederzeit und schnell gerade in diesen Verfahren wiederfinden und verstetigen, was einen affirmativen Bezug auf sie notorisch erschwert. Historisch betrachtet ist dieses Argument ein Dauerbrenner. ${ }^{12}$

9 Vgl. nach J. Raz etwa S. Wall, Liberalism, Perfectionism and Restraint [1998], Cambridge ${ }^{2} 2007$.

10 So kann er an den Rändern der Gerechtigkeitstheorie verortet werden; vgl. R. Dworkin, Sovereign Virtue. The Theory and Practice of Equality, Cambridge/Mass. 2000, $281 \mathrm{f}$. S. Jentsch (Chancengleichheit und Perfektionismus. Zur Unerlässlichkeit einer Theorie des Guten im Bereich des Gerechten, Paderborn 2009, 123) verortet den Perfektionismus in der „Rechtskonkretisierung“ (anstelle seiner Begründung oder Anwendung). Im vorliegenden Beitrag hat sie ihre Position radikalisiert und problematisiert die Neutralität (vgl. dies., Perfektionismus und soziale Gerechtigkeit, in: Deutsche Zeitschrift für Philosophie, 58 (2010) (vorliegendes Heft), Abschnitt III).

11 Vgl. G. Sher, Beyond Neutrality: Perfectionism and Politics, Cambridge 1997; vgl. auch C. Henning, Natur und Freiheit im Perfektionismus, in: Deutsche Zeitschrift für Philosophie, 58 (2010) (vorliegendes Heft), Abschnitt I; zu älteren Versionen vgl. ebd., Abschnitt III; sowie A. Rüdiger, Produktive Negativität, a. a. O., Abschnitt III. Auch Rawls' spätere Idee eines „überlappenden Konsenses“ idealisiert: De facto gingen die „constitutional essentials“ (Justice as Fairness: A Restatement, Cambridge 2001,28) dem Konsens über die Verfassung in den USA voran, und kamen keineswegs konsensual zu Stande (die Federalist Papers wurden ja als Abweichung hervorgebracht). Man bemerke übrigens die semantische Nähe des Begriff ,freestanding“ (J. Rawls, Political Justice, New York 1993, 374 f.; auch bei Nussbaum, vgl. S. Jentsch, Perfektionismus und soziale Gerechtigkeit, a. a. O.) mit Karl Mannheims umstrittener Idee „freischwebender“ Intellektueller.

12 Man denke an die Kritik des Geldes von Aristoteles bis Marx, die sich noch bei H. Croly oder M. Nussbaum findet: Geld als Prototyp eines ,rein‘ formalen Mediums hängt, glaubt man G. Simmel, mit den liberalen Begriffen von (wählendem) Individuum und (Wahlfreiheit gewährender) Gesellschaft zusammen (vgl. G. Simmel, Philosophie des Geldes, Leipzig 1900, Kap. 6). Doch ist Geld damit gerade nicht „,neutral“: Es kann höchst negative Folgen haben (etwa eine Entqualifizierung 
Das Bild ändert sich vollends, wenn die Alternative zum Guten und seiner Förderung nicht das Neutrale (im schlimmsten Fall eine Unterlassung), sondern das Schlechte ist. Was, wenn eine in der Rechtfertigung neutral sein wollende Politik schlechte Folgen hat? Nehmen wir an, die Devise, jeder Mann könne mit ,seiner' Frau tun, was er will (also ohne eine partikulare Moral vorzuschreiben), sei schlecht für Frauen und Kinder, und damit letztlich auch für die Männer. Muss man hier nicht die Integrität der Frauen schützen - selbst wenn manche Männer es nicht wollen? Warum dann nicht gleich das Gut einer selbstbestimmten Partnerschaft fördern? ${ }^{13}$ Und da das in einigen Ländern ohnehin - zumindest ansatzweise - getan wird, warum eine Philosophie vertreten, die nicht denken kann, was in der Praxis längst üblich ist? Seltsamerweise tut sich der Perfektionismus in , gegenwärtigen Debatten " schwer damit, dem Dualismus der liberalen Neutralität etwas entgegenzusetzen. Dabei ist diese Idee keine überhistorische Gegebenheit, sondern eher jungen Datums ${ }^{14}$, während perfektionistische Gegenpositionen auf eine lange Geschichte zurückblicken können - nur sind sie in der Debatte wenig präsent und vielleicht daher Verdächtigungen ausgesetzt. Daher ist ein Anliegen dieses Schwerpunkts, die Traditionen auf solche Fragen hin neu zu lesen.

Der zweite Gegenwind bläst dem Perfektionismus in Sachen Sozialtheorie entgegen (2). Wer an guten Folgen für die menschliche Entwicklung interessiert ist, sollte über kontrollierbares Wissen von diesen Folgen verfügen. Ein solches lässt sich aber ,rein " philosophisch nicht haben; es bedarf des Blicks auf benachbarte Sozialwissenschaften. (Damit ist bereits benannt, was Ulrich Steinvorths Beitrag mit dem aus seiner Sicht perfektionistisch unterfütterten Liberalismus John Lockes anstellt: Er konfrontiert ihn mit veränderten sozialtheoretischen Befunden und zieht so aus Lockes Vorgaben andere Konsequenzen als jener. ${ }^{15}$ War eine solch disziplinäre Offenheit für Autoren von Condorcet und Adam Smith bis zu Mill und Marx selbstverständlich, droht ihm heute der wohlfeile Vorwurf eines naturalistischen Fehlschlusses.

Die gegenläufige Gefahr „freistehender“ philosophischer Rechtfertigungen ist allerdings, dass sie in ausgedachten Welten triftig sein mögen, nicht aber in der realen. Ist das vorzuziehen? Man könnte zugespitzt sagen, dass eine solch ausgedachte Welt die Grundlage des neutralistischen Liberalismus bildet - sofern er sich nämlich auf ein idealisiertes Bild des Marktes stützt, in dem Menschen zum ,wechselseitigen Vorteil“ effizient und gerecht miteinander „kooperieren“ und immer schon reziproke Normen verinnerlicht haben. Natürlich würde jeder ,Eingriff‘ diese schöne Welt stören. Doch eine solche Welt gibt es nur in Lehrbüchern. Die Frage, ob Normen, die auf diesem Bild aufruhen - wie attraktiv sie sein mögen -, für die Gegenwart tatsächlich unterstellt und gegen Theorien des Guten gehalten werden können, ist aus perfektionistischer Sicht keine prinzipielle, sondern eine empirische. Das meint weniger Befragungen über das moralische Denken der Menschen wie bei David Miller, sondern eher Erkenntnisse über die wirkliche Beschaffenheit sozialer Systeme und ihren Einfluss auf die menschliche Entwicklung. Um ein Standardbeispiel zu bringen: Ein Wert wie Freiheit ist

sozialer Beziehungen oder eine Förderung von Korruption, setzt man es - zum Beispiel in der Entwicklungshilfe - im Gießkannenprinzip ein). Eben das macht eine Besinnung darauf nötig, welche Ziele („Güter") mit diesem scheinbar ,reinen“ Mittel eigentlich angestrebt wurden.

13 Vgl. K. Yuracko, Perfectionism and Contemporary Feminist Values, Bloomington 2003.

14 Vgl. M. Sandel, Democracy's Discontent: America in Search of a Public Philosophy, Cambridge/ Mass. 1996, $274 \mathrm{ff}$.

15 Vgl. U. Steinvorth, Locke, Arbeit und Emanzipation, in: Deutsche Zeitschrift für Philosophie, 58 (2010) (vorliegendes Heft). Eine indirekte Beziehung Lockes zum Perfektionismus (neben der von Steinvorth beanspruchten direkten über den Arbeitsbegriff) läuft über seinen radikalen Sensualismus: Dieser führte etwa bei Condorcet zur Überzeugung einer natürlichen Gleichheit und damit zur Perfektibilität (vgl. C. Henning, Natur und Freiheit im Perfektionismus, a. a. O., Abschnitt II). 
wenig , wert', solange in der Realität nur eine Minderheit in seinen Genuss kommt. Statt ihren abstrakten Wert stets erneut hervorzuheben, kann es der Entwicklung der Menschen dienlicher sein, ihr praktisch unter die Arme zu greifen. ${ }^{16}$

Dennoch trifft der Vorwurf des naturalistischen Fehlschlusses einen Punkt: Sozialtheorie allein kann schwerlich sagen, was ,gut“ ist. Im Perfektionismus kommen an dieser Stelle oft Theorien über die Natur des Menschen ins Spiel (3). Doch auch das bringt ihm Gegenwind ein: Dies sei „essentialistisch“ oder naturalistisch und daher nicht mit dem Stand gegenwärtiger Debatten vereinbar (obwohl Pluralismus, wenn er ein Wert ist, dies allemal für ,Debatten' sein müsste). Diese Sicht teilen auch einige neuere Perfektionisten. Sie ist allerdings nicht zwingend, sondern beruht auf einer speziellen Lesart insbesondere von Aristoteles: $\mathrm{Zu}$ Grunde liegt ihr die Vorstellung, Theorien, die der menschlichen Natur eine Idee vom Guten entnehmen, basierten auf einer „metaphysischen Biologie“.${ }^{17}$ So verstanden, scheinen Sätze über die menschliche Natur die menschlichen Möglichkeiten einzuschränken und auf wenige Varianten zu sein festzulegen. ${ }^{18}$ In der Tat haben sich konkrete ,Ableitungen“ richtiger Lebensformen allzu häufig als bloße Immunisierung kontingenter Vorurteile (gegen Frauen, Schwarze etc.) - und damit als falsch - erwiesen. Die anti-essentialistische „Hermeneutik des Verdachts“ (Paul Ricœur) speist sich damit nicht zuletzt aus einer Sorge um die individuelle Freiheit. Nicht ganz zu Unrecht: Noch jüngst wurde ja geurteilt, Homosexualität sei gegen die menschliche Natur und daher abzulehnen. Darüber spielte sich vor Gericht eine Debatte zwischen Philosophen ab, in der man sich nicht einigen konnte. ${ }^{19}$ Das dürfte nicht sein, wäre alles so einfach wie die hermetische Lesart der ,metaphysischen Biologie“ unterstellt.

Nun muss man diese hermetische Lesart nicht teilen. Obwohl Aristoteles auf Grund seiner Ausgrenzung von Frauen und Nichtbesitzenden problematisch ist, lässt sich seine anthropologische Ethik - der Nukleus perfektionistischen Denkens - auch pluralistisch lesen: Menschliche Potenziale werden durch eine kontingente Praxis des Einübens verwirklicht, die von der Kultur und den Entscheidungen Einzelner abhängig bleibt. Es gibt folglich nicht den einen besten Weg, sondern eine Plastizität des Potenzials und eine Pluralität von Entwicklungsmöglichkeiten. ${ }^{20}$ Folglich widersprechen weder Kontingenz noch Wandel dieser Konzeption. Doch

16 Daher enthält etwa J. Deweys Ethik lange sozialtheoretische Passagen, die in der Forderung nach Ermöglichung „effizienter“ (sprich positiver) Freiheit gipfeln (vgl. C. Henning, Natur und Freiheit im Perfektionismus, a. a. O., Abschnitt III). Oben gemeint ist: D. Miller, Grundsätze sozialer Gerechtigkeit [1999], Frankfurt/M. 2009.

17 A. MacIntyre, After Virtue, Notre Dame 1984, 163; zitiert unter anderem bei: G. Sher, Beyond Neutrality, a. a. O., 226; ähnlich bereits: J. Dewey, Human Nature and Conduct [1922], Amherst 2002, 174.

18 I. Berlin, der diese kritische Sicht etabliert hat (,there can [...] be only one correct way of life [...] The unwise must be dragged towards it"), spricht allerdings selbst affirmativ von einer „essence of human nature" (ders., Two Concepts of Liberty [1958], in ders., Liberty, Oxford 2002, 197 f., 173, vgl. 183: ,the essence of men is that they are autonomous beings").

19 J. Finnis u. M. Nussbaum, Is Homosexual Conduct wrong? A Philosophical Exchange, in: New Republic, 15 (1993), 12-13. Sher (Beyond Neutrality, a. a. O., 216 f.) zum Beispiel ist hier eher liberal, die katholische Kirche hingegen nimmt in diesem Punkt eine naturalistische Engführung vor: Sexualität diene nur dem natürlichen Zweck der Fortpflanzung. Das ist - sogar aus Sicht der Naturwissenschaften - schwer nachvollziehbar.

20 „In the case of practical sciences [...] Aristotle held that the movement or development of their subject matters cannot be accounted for by this simple scheme of the actualization of inherent potentialities. Rather, an intermediate concept must be invoked: habit or habituation (hexis or ethos), which reflects human choices and conditionings [...] these potentialities can be turned in many different directions [...] It is thus a mistake to interpret Aristotle's doctrine of the human good along the lines of his ,metaphysical biology', as if that good were a ,natural end" or telos." (A. Gewirth, Self-Ful- 
bleibt eine Differenz sowohl zum Subjektivismus, der nur die individuelle Wahl kennt, wie zum Kommunitarismus, der sich nur auf die Kultur stützt: Es wird ein Potenzial verwirklicht - oder eben nicht. Wenn es verwirklicht wird, macht sich das als Gedeihen (,flourishing“) bemerkbar; eine verhinderte Entwicklung hingegen kann als Entfremdung oder Anomie diagnostiziert werden. Zwar muss sich die Theorie, da es keinen unmittelbaren Zugang zum Potenzial gibt, auf Kulturen und Personen beschränken, in denen es beispielhaft entwickelt wurde. (Das schließt neue ,experiments in living“ keineswegs aus.) Gerade die leibliche Verankerung erlaubt es allerdings, auszumachen, welche Praxen der individuellen Entwicklung ,gut" tun und welche weniger. ${ }^{21}$ Die menschliche Natur schreibt aus dieser Sicht also keine bestimmte Art zu leben vor, vermag aber in Vergleichen zumindest grob anzuzeigen, welche Arten zu leben ihr förderlich sind. Die einzige Teleologie, die darin liegt, ist die Vorstellung, dass das menschliche Leben an ein nachhaltiges Gelingen verwiesen ist. Davor muss sich niemand fürchten, und das nimmt der postmodernen Humanismus-Phobie ihr Feindbild. Vielleicht eignet sich diese Konzeption sogar besser als jene Vermeidungsstrategie, illiberalen Anthropologien entgegenzutreten (etwa vorgestrigem Naturrecht oder ultra-liberalen enhancement-Prophetien) ${ }^{22}$ : nicht durch Ächtung des Themas, sondern durch einen eigenen, emanzipatorischen Begriff der menschlichen Natur.

Damit wurden in aller Kürze drei Felder genannt, auf denen der Perfektionismus sich philosophisch zu bewähren hat. Man könnte nun die Diagnose stellen, dass er dabei solange schlecht fährt, wie er das reichhaltige Reservoir an Argumenten verschmäht, das in der perfektionistischen Tradition bereitsteht. Erstaunlich an gegenwärtigen Entwürfen ist nämlich vor allem die merkwürdige Geschichtsvergessenheit, mit der perfektionistische Inhalte reformuliert werden. Erstaunlich ist dies deswegen, weil das politische Denken seit Platon und Aristoteles oft perfektionistische Züge dieser oder jener Spielart hatte. Nicht alle davon sind der Mottenkiste freiheitsfeindlichen Denkens zu überlassen; jedenfalls nicht mehr, als einige Zweige ,rein' liberalen Denkens es verdient hätten. Diese Traditionen sollten solange nicht vergessen werden, wie sie noch heute aufschlussreiche Einsichten aufweisen; und dieser „Horizontverschmelzung" möchte dieser Schwerpunkt dienen.

Damit genug der erläuternden Worte und noch ein Blick auf die Autoren dieses Schwerpunktes: Ulrich Steinvorth, in der deutschsprachigen politischen Philosophie seit langem eine feste Größe, hat den Perfektionismus schon vor Jahren in sein Denken einbezogen und nicht als Gegensatz, sondern als Voraussetzung des Liberalismus behandelt. ${ }^{23}$ Diesen Gedanken

fillment, Princeton 1998, 11 f.; ähnlich: T. Rentsch. Konstitution der Moralität, Frankfurt/M. 1990, 290 f.; sowie: J. McDowell, Wert und Wirklichkeit: Aufsätze zur Moralphilosophie, Frankfurt/M. 2002, 13, Vorrede der Herausgeber; 60 f.)

21 Mit ihrem bewertenden Kulturvergleich setzen die Glückswissenschaften daher fort, was Aristoteles begann. Dieser verglich Verfassungen, und noch der Zweck von M. Nussbaums capability approach bestand darin, Vergleichskriterien für gute Entwicklungspolitik zu liefern. Da solche Vergleiche anfällig sind für „kolonisierendes“ Denken, plädiert Nussbaum für partizipative und korrigierbare Ansätze (zur Glücksforschung vgl. neuerdings: Glück: Ein interdisziplinäres Handbuch, hg. v. D. Thomä u. a., erscheint bei Metzler).

22 Vgl. etwa A. Buchanan, Human Nature and Enhancement, in: Bioethics, 23 (2008), 141-150.

23 Die Gerechtigkeit von Institutionen könne ,nicht ohne Rücksicht darauf gemessen werden, ob sie zur Entwicklung bestimmter Eigenschaften (,Vollkommenheiten') beitragen. [...] Denn die Fähigkeit, über sich selbst zu verfügen und dabei Gründen zu folgen, in deren Betätigung alle politische Freiheit besteht, ist eine Eigenschaft oder Vollkommenheit, die entwickelt werden muss, bevor sie betätigt werden kann.“ (U. Steinvorth, Gleiche Freiheit: Politische Philosophie und Verteilungsgerechtigkeit, Berlin 1999, 156, vgl. 275 ff.) 
wendet er im vorliegenden Beitrag kritisch auf J. Locke an: Man könnte seine Überlegungen so zuspitzen, dass ein Liberalismus, der von Voraussetzungen lebt, die er nicht garantieren kann, keiner - oder ein nicht zu Ende gedachter - ist. Nicht zufällig kommen daher ursprünglich perfektionistische Werte des Liberalismus, etwa die Autonomie durch selbstbestimmte Tätigkeit, heute vor allem bei Kritikern der überkommenen Arbeitsgesellschaft und Befürwortern eines bedingungslosen Grundeinkommens zum Zuge, zu denen sich auch Steinvorth gesellt. ${ }^{24}$

Axel Rüdiger konnte bereits in seiner Arbeit über Halle als Zentrum der Aufklärung die Entwicklung von Ideen institutionengeschichtlich verankern. ${ }^{25}$ Sein Beitrag beleuchtet die Entwicklung des perfektionistischen Denkens in einem durch Kants Kritik noch immer verfemten Feld: der politischen Theorie der deutschsprachigen Aufklärung. Ironischerweise erlaubt es gerade M. Foucaults kritischer Blick, der noch in liberalen „Regimen“ Techniken der Steuerung von Menschen freizulegen vermag ${ }^{26}$, im Umkehrschluss in ,vorliberalen Regimen auch Mechanismen einer Freiheitsermöglichung zu erblicken. (Diese trugen ihre Früchte allerdings erst später.) Rüdiger kann jedenfalls zeigen, dass der normative Kern des Aufklärungsdenkens - welches ja auch die „Policeywissenschaften“ ins Leben rief - perfektionistisch ist, also an einer guten Entwicklung der Individuen interessiert ist. Der Beitrag unterscheidet eine Palette verschiedener Varianten dieses Denkens ${ }^{27}$; auch solche, die bis in den Deutschen Idealismus, ja bis heute wirken. Er nutzt dabei ,postmoderne' Einsichten als Analyseraster, statt sie, wie zuweilen üblich, schlicht gegen ältere Denkgebäude zu stellen. Nimmt man dies zur Kenntnis, müsste eine Kritik am Perfektionismus zunächst konkretisieren, welche Variante überhaupt gemeint ist.

Der Deutsche Idealismus wird im Beitrag von Michael Schefczyk in Gestalt Hegels näher untersucht, von dem eine der wirkmächtigsten Varianten der Idee der „Selbstverwicklung“ stammt. Die Gefahr, die Schefczyk nicht nur bei Hegel, sondern auch bei der Reaktualisierung Hegels durch Michael Theunissen freilegt, besteht darin, dass das Individuum sich hier nur als Repräsentant des Allgemeinen, und damit gerade nicht als Individuum, verwirklicht. Schefczyk hat sich in jüngster Zeit mit J. S. Mill beschäftigt, welcher unter die liberalen Perfektionisten zu rechnen ist. ${ }^{28}$ Hier zieht er in einem dritten Schritt einen Zeitgenossen Mills, den Britischen Idealisten Th. H. Green (1836-82), in seine Betrachtung ein. Obwohl dieser die Idee der Selbstverwirklichung letztlich moralistisch überfrachte, gelinge es seiner Konzeption durch ihre offenere Metaphysik und Sozialtheorie besser als Hegel, dem „Recht auf Besonderheit" des Individuums gerecht zu werden.

24 Vgl. bereits U. Steinvorth, The Right to work and the Right to develop One's Capabilities, in: Analyse und Kritik, 101-113 (2009), 101-114. Schon André Gorz hat seine Position von einem Recht auf Arbeit zu einem solchen auf ein Grundeinkommen radikalisiert und dabei perfektionistisch argumentiert: „Die volle Entfaltung jedes einzelnen wird zum Ziel, das die Gesellschaft ermöglichen muss, und das selbst eine Gesellschaft ermöglicht, wo die Sorge um die freie Entfaltung der Persönlichkeit gesellschaftlich anerkannt und legitimiert wäre.“ (A. Gorz, Arbeit zwischen Misere und Utopie, Frankfurt/M. 2000, 94)

25 A. Rüdiger, Staatslehre und Staatsbildung. Die Staatswissenschaft an der Universität Halle im 18. Jahrhundert, Tübingen 2005.

26 Aufgegriffen zum Beispiel in: M. Bohlender, Metamorphosen des liberalen Regierungsdenkens. Politische Ökonomie, Polizei und Pauperismus, Weilerswist 2007.

27 Vgl. schon C. Schwaiger, Vollkommenheit als Moralprinzip bei Wolff, Baumgarten und Kant, in: Vernunftkritik und Aufklärung. Studien zur Philosophie Kants und seines Jahrhunderts, hg. v. M. Oberhausen, Stuttgart 2001, 312-328.

M. Schefczyk u. D. Kuenzle, John Stuart Mill zur Einführung, Hamburg 2009. 
Der Beitrag von Christoph Henning entwirft einen Begriff der menschlichen Natur, der dem Essentialismus-Vorwurf entgehen kann. Wie eine Analyse George Shers exemplarisch zeigt, ist ein solcher deswegen nötig, weil sein Fehlen den Perfektionismus blass und inkonsistent aussehen lässt. Er scheint in eine Falle getappt zu sein: Entweder meint er auf einen Begriff der menschlichen Natur verzichten zu müssen, kommt dann aber kaum zu konkreten normativen Bestimmungen, oder er legt derart reduzierte Naturbegriffe zu Grunde, dass ein Individualismus damit nur schwer vereinbar ist. ${ }^{29}$ Ein Ausweg aus diesem Dilemma wird hier zunächst Condorcet, der mit Rousseau als Denker der perfectibilité gilt ${ }^{30}$, und dann dem US-amerikanischen Progressivismus entnommen (vor allem J. Dewey). Die zukunftsoffene Sichtweise dieser Denker birgt einen anschlussfähigen Begriff der menschlichen Natur. Dieser ,progressive“ Begriff (der bei Dewey von Green inspiriert ist) legt die Menschen weder auf eine bestimmte Teleologie fest, noch stellt er sie in unverbindliche Beliebigkeit. Mit ihm lassen sich klare Bedingungen für eine gute Entwicklung des Menschen benennen.

Sabine Jentsch schließlich, die jüngst eine perfektionistische Auslegung und Verteidigung der Chancengleichheit vorgelegt hat ${ }^{31}$, konfrontiert in ihrem Beitrag den aus ihrer Sicht zu minimalistisch ansetzenden Perfektionismus Martha Nussbaums mit Gerechtigkeitstheorien von John Rawls und Hillel Steiner. Ausgangspunkt ihrer Überlegungen ist die Frage, welche Konzeption es am besten erlauben würde, auch Menschen mit Behinderungen als Statusgleiche in den Geltungsbereich der Gerechtigkeitstheorie einzubeziehen. ${ }^{32}$ Dabei drängt sie darauf, dass Nussbaums Rawls-kritisches Postulat einer Inklusion möglichst aller Menschen noch der Begründung harrt, welche sie selbst in dem Konzept einer moralischen Gleichheit in der Verschiedenartigkeit findet. ${ }^{33}$ Damit ist eine der vielen Stellen benannt, an denen perfektionistische Theorien in gegenwärtigen Debatten mehr und mehr relevant werden.*

Christoph Henning, St. Gallen

29 „This idea of individual perfection [...] does not have plausible consequences.“ (T. Hurka, Perfectionism, a. a. O., 15) Man denke auch an die Beschneidung einiger individueller Freiheiten im katholischen „Naturrecht“.

30 Das Wort taucht 1750 bei Turgot auf; vgl. Historisches Wörterbuch der Philosophie, Bd. VII, 239.

31 Vgl. S. Jentsch, Chancengleichheit und Perfektionismus, a. a. O.

32 Mit ähnlicher Thematik: C. Lowry, Perfectionism within Neutrality: Extending Liberalism to Citizens with Disabilities, $\mathrm{PhD}$ thesis 2008, Kingston/Canada.

33 Um ein ähnliches Konzept kreisen verschiedene Beiträge: So hat bereits der bei A. Rüdiger behandelte Pufendorf die Gleichheit in die gleichen moralischen Vermögen gelegt (vgl. K. Saastamoinen, Pufendorf on Natural Equality, Human Dignity, and Self-Esteem, in: Journal of the History of Ideas, 71 (2010), 39 ff.). Zwar könnte auch dieses Kriterium, etwa bei geistiger Minderbegabung, wieder abgesprochen werden, was Jentschs „Statusgleichheit“" zuwiderliefe (,moralische Gleichheit" meint also Objekte, nicht nur Subjekte der Moral). Das würde einen inklusiveren Gleichheitsbegriff erfordern - vielleicht einen, wie ihn Henning bei Condorcets ,natürlicher Gleichheit“ in der ersten Natur vermutet, die sich qua Gattungszugehörigkeit auf alle Menschen erstreckt? Dann ließe sich, wie es auch das von Steinvorth unterstützte Grundeinkommen vorsieht, eine Zusprache gleicher Rechte (auch sozialer) nicht länger von speziellen Kriterien, etwa der vollen Rationalität oder der Arbeitsfähigkeit beziehungsweise Arbeitswilligkeit, abhängig machen.

* Zuletzt sei dem Schweizerischen Nationalfonds (SNF) ein Dank für die Förderung ausgesprochen. 